EPJ Web of Conferences 75, 04012 (2014)

DOI: $10.1051 /$ epjconf/ 20147504012

(C) Owned by the authors, published by EDP Sciences, 2014

\title{
An atomic force microscopy study of Eurofer-97 steel
}

\author{
D. Stamopoulos ${ }^{1, \mathrm{a}}$, E. Aristomenopoulou ${ }^{1}$, M.J.R. Sandim ${ }^{2}$, H.R.Z. Sandim ${ }^{2, \mathrm{~b}}$ and M. Pissas ${ }^{1}$ \\ ${ }^{1}$ Institute of Advanced Materials, Physicochemical Processes, Nanotechnology and Microsystems, National Center for \\ Scientific Research 'Demokritos', 153 10, Aghia Paraskevi, Greece \\ ${ }^{2}$ Escola de Engenharia de Lorena, Universidade de São Paulo, 12602-810, Lorena-SP, Brazil
}

\begin{abstract}
In recent years the microstructure, mechanical and magnetic properties of Eurofer-97 steel are studied intensively due to its application in nuclear fusion power plants. Its microstructure is usually accessed by means of electron microscopy. Here we present an alternative approach utilizing Atomic Force Microscopy (AFM) to study as-received Eurofer-97 steel. We recorded both the Height Signal (HS) and Phase Signal (PS) that provided information on the morphologic and inelastic topography, respectively. With the HS we detected spherical particles (SPs) of size 50-2000 $\mathrm{nm}$. Interestingly, micrometer SPs $(0.1-2.0 \mu \mathrm{m})$ are randomly distributed, while nanometer SPs $(50-100 \mathrm{~nm})$ are sometimes arranged in correlation to grain boundaries. The PS clearly revealed that the micrometer SPs exhibit inelastic properties. Though we cannot identify the elemental composition of the SPs with AFM, based on relevant electron microscopy data we ascribe the nanometer ones to the $\mathrm{TaC}$, TiN and $\mathrm{VN}$ and the coarse micrometer ones to $\mathrm{M}_{23} \mathrm{C}_{6}(\mathrm{M}=\mathrm{Cr}, \mathrm{Fe})$. The latter class of SPs can probably be active sites that influence the mechanical properties of Eurofer-97 steel upon annealing as observed in relevant electron microscopy based studies.
\end{abstract}

\section{Introduction}

In recent years the mechanical and magnetic properties of ferritic-martensitic Eurofer steels, either Eurofer-97 or ODS-Eurofer, have been intensively studied since they are potential candidates for application in nuclear fusion power plants $[1,2]$. Furthermore, the investigation of the microstructure of these steels is important because their mechanical and magnetic properties are influenced by even minor structural differences. Until now, the microstructure of Eurofer-97 steel has been mostly investigated by means of electron microscopy [1-4].

Here we present an alternative approach with Atomic Force Microscopy (AFM) [5], a technique that is used in a wide spectrum of applications in physics, materials science, biophysics etc [6-10]. By using AFM here we study both the morphologic and inelastic topographies of as-received Eurofer-97 steel. We detected circular particles of nanometer and micrometer size that by comparing to the electron microscopy data $[1,2]$ can be ascribed to $\mathrm{TaC} / \mathrm{TiN} / \mathrm{VN}$ and $\mathrm{M}_{23} \mathrm{C}_{6}(\mathrm{M}=\mathrm{Cr}, \mathrm{Fe})$, respectively. Interestingly, the micrometer SPs exhibit inelastic properties that are different from those of the surrounding $\mathrm{Fe}-\mathrm{Cr}$ matrix. These results indicate that the $\mathrm{M}_{23} \mathrm{C}_{6}(\mathrm{M}=\mathrm{Cr}, \mathrm{Fe})$ particles could probably play a role in the mechanical properties of Eurofer-97 steel upon annealing [1-4].

\footnotetext{
Corresponding authors: ${ }^{\text {a }}$ densta@ims.demokritos.gr

b hsandim@demar.eel.usp.br
}

\section{Methods}

\subsection{Material}

The as-received Eurofer-97 sample has the nominal composition $9 \mathrm{Cr}-1.1 \mathrm{~W}-0.125 \mathrm{Ta}-0.25 \mathrm{~V}-0.105 \mathrm{C}-0.6 \mathrm{Mn}-$ $0.036 \mathrm{~N}$ (wt.\%) and a fully bcc ferritic structure [1]. The sample was polished initially with a diamond paste and finally with colloidal silica of $0.06 \mu \mathrm{m}$ radius spherical particles. No further chemical etching was used.

\subsection{Technique}

The AFM experiments were conducted by means of a scanning probe microscope Solver PRO [NT-MDT Co, Moscow, Russia] having a 100x100x5 $\mu \mathrm{m}^{3}$ xyz-scanner hosted on an active vibration isolation table [Halcyonics MOD-1M Plus]. Measurements were performed in the semi-contact mode with NCHR probes that end to silicon nitride tips [Nano and More GmbH, Wetzlar, Germany] of nominal parameters, tip radius below $12 \mathrm{~nm}$, spring constant $=42 \mathrm{Nm}^{-1}$ and resonance frequency $=320 \mathrm{kHz}$. The optimum imaging results were obtained for scanning parameters within: line-scanning-frequency $=1-2 \mathrm{~Hz}$, area-scanning-size $=0.5 \times 0.5-50 \times 50 \mu \mathrm{m}^{2}$ and lines-perimage $/$ points-per-line $=256-512$.

In the present experiments we recorded both the Height Signal (HS) that refers to the morphologic topography (mapping of the spatial relief of the sample 
surface) and the Phase Signal (PS) that refers to the inelastic topography (mapping of sample surface areas exhibiting adhesion and/or viscoelastic properties). Below let us address the basics of the employed procedure for the non-expert readers.

The AFM technique is based on mechanical means that is on scanning the sample surface, xy-plane, with a special probe: a sharp tip (typical end radius: a few $\mathrm{nm}$ to a few tens of $\mathrm{nm}$ ) is realized at the end of a micrometer cantilever (typical dimensions: length a few hundred $\mu \mathrm{m}$, width a few tens $\mu \mathrm{m}$ and thickness a few $\mu \mathrm{m}$ ) that protrudes at the one side of an orthogonal holder chip (typical dimensions: a few $\mathrm{mm}$ ). A representative probe used in our experiments, is shown in figures 1(a)-1(c). In figure 1(a) we show an optical microscopy image of the complete probe in side view, while in figures $1(\mathrm{~b})$ and 1(c) we gradually focus on the tip, in front view, by using optical and electron microscopy, respectively. Evidently, the specific probe is quite contaminated with stray material and structurally degraded due to extended use.

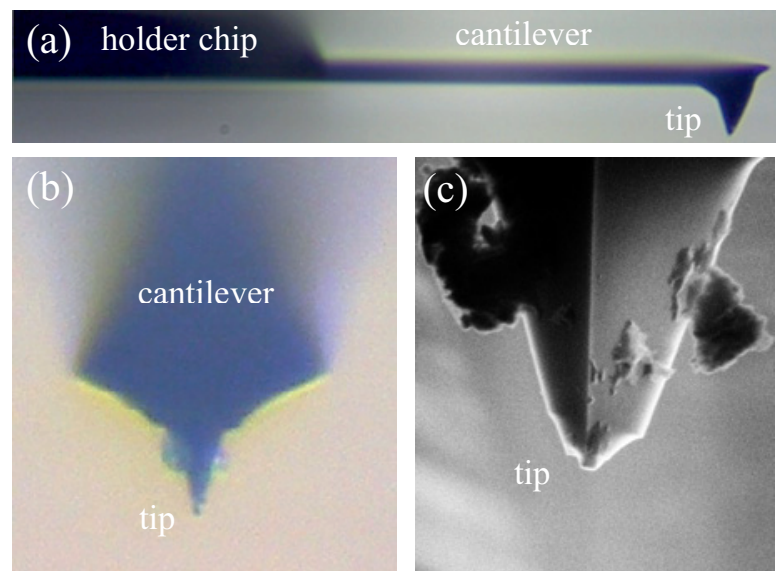

Fig. 1. (a) Optical microscopy image (side view) of the complete probe, holder chip-cantilever-tip (x200). (b) Optical microscopy image (front view) of the cantilever-tip (x400). (c) Scanning electron microscopy image (front view) focused on the same tip (x20000).

The scanning progresses on the xy-plane in the socalled 'raster pattern' that is in a sequence of equidistant parallel lines, each one of them divided in equidistant points; the overall array of distinct points resulted on the xy-plane follows a 'grid pattern'.

AFM operation can be classified in two basic modes, static and dynamic. Specifically, while scanning the cantilever can be (i) static; the tip is in continuous contact with the sample surface and (ii) oscillating; the tip is in (ii.a) instantaneous semi-contact with the sample surface at the lowest position of the oscillation cycle or (ii.b) non-contact with the sample surface during the complete oscillation cycle. In dynamic modes (ii.a) and (ii.b) the cantilever is forced to oscillate at its resonance frequency around an equilibrium z-position above the sample surface, with appropriate amplitude of a few tens of nanometers. The external sinusoidal driving force of appropriate amplitude and frequency is produced by a piezoelectric base on which the complete probe is mounted [10-12].
Since in this work we employed the semi-contact mode below we provide details on this specific operation. In the semi-contact mode, after the tip-sample engagement ('landing' of the tip onto the sample surface), the oscillation characteristics of the cantilever, that is amplitude and phase, are the signals of interest that are recorded during scanning the sample surface [10-12]. Subsequently, each one of these two signals can be used as a feedback parameter to control the process. In our measurements we used the oscillation amplitude, thus below we focus on this operation. The oscillation amplitude is recorded by a quartet of semiconductor photo-detectors, in square array configuration, that track the deflection of the oscillating spot of a laser beam while the latter is reflected at the back side of the oscillating cantilever. Accordingly, the oscillation amplitude of the cantilever is used as a Feedback Signal (FS) that is compared to a prefixed reference value, the so-called Set Point (SP) that ranges within a percentage (usually within $20-80 \%$ ) of the free-space oscillation amplitude of the cantilever that is prior to tip-sample engagement. The resulted oscillation amplitude, after tip-sample engagement, corresponds to a few tens of nanometers. The subtract signal FS-SP is an error signal that should be kept zero during scanning to control the process.

However, during scanning on the xy-plane, the tipto-sample distance (z-axis) changes due to the unknown spatial variation of the sample surface that is to be surveyed. Since the interaction between the tip and the sample depends on their distance, the oscillation amplitude of the cantilever tends to change when a $\mathrm{z}$-axis variation of the sample surface is experienced. To maintain the oscillation amplitude of the cantilever constant, the stage where the cantilever is mounted readjusts its vertical position (along z-axis) accordingly, that is by an amount equal to the $\mathrm{z}$-axis variation of the sample surface experienced at each point of the 'grid' pattern'. This z-axis readjustment is used to produce a digital reconstruction of the xyz morphologic topography of the sample surface that is the HS image.

As mentioned above, except for the oscillation amplitude of the cantilever, the oscillation phase can be recorded simultaneously [10-12]. The oscillation phase depends on the specific interaction of the tip with the sample and is mostly affected by inelastic processes such as adhesion and viscoelasticity [13-16]. Thus, the respective image of the spatial variation of the oscillation phase, that is the PS image, is termed inelastic topography. Accordingly, the PS image can be used to distinguish areas of different composition that otherwise are coplanar along the z-axis, thus they are indistinguishable in the respective HS image [13-16].

\section{Results}

In figure 2(a) we present the HS of an $11 \times 11 \mu \mathrm{m}^{2}$ area that surprisingly resembles electron microscopy images that are usually obtained in these materials [2]. We stress that the HS detects nano/micro-size grains exhibiting nm differences in height (z-axis) as illustrated by the colorcoding bar on the right side of the image. 

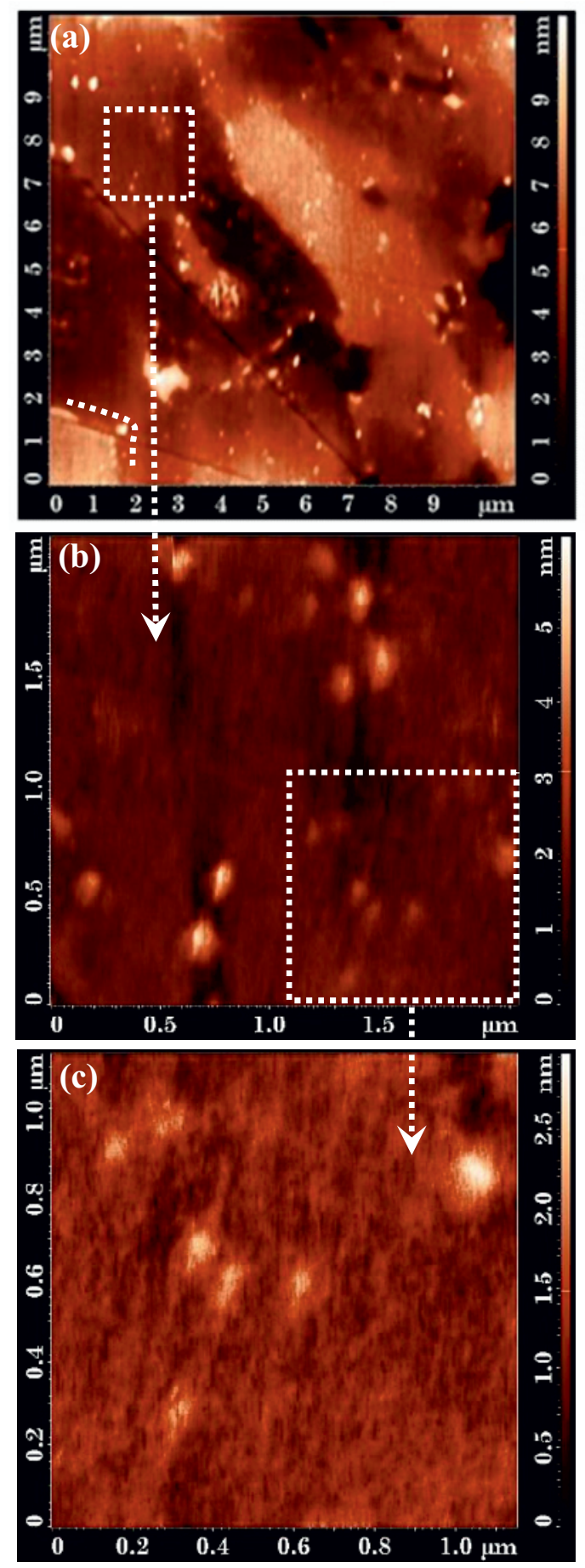

Fig. 2. Representative AFM images of the morphologic topography that is the HS of (a) an $11 \times 11 \mu \mathrm{m}^{2}$ area, (b) an $2 \times 2$ $\mu \mathrm{m}^{2}$ first sub-area and (c) an $1.2 \times 1.2 \mu \mathrm{m}^{2}$ second sub-area as indicated by the dotted rectangles.

Also, coarser spherical particles (SPs) of diameter 50-2000 $\mathrm{nm}$ are detected, while a couple of straight indentations/grooves, possibly produced by the polishing process, are observed. Interestingly, micrometer SPs, 0.1$2.0 \mu \mathrm{m}$ are randomly distributed, while nanometer SPs are sometimes arranged in correlation to grain boundaries (see dotted curve in the lower left corner of figure 2(a)).

Continuing, in the HS figures 2(b) and 2(c) we have progressively focused on sub-areas indicated by the white-dotted rectangles for the better observation of the nanometer features. The frame-to-frame reproducible detection of the SPs observed in figures 2(b)-2(c) validates their existence. Moreover, the size of the SPs detected in figure 2(c) is determined to range within 50$100 \mathrm{~nm}$. These nanometer SPs can be ascribed to the $\mathrm{TaC}$, TiN and $\mathrm{VN}$ fine particles that are typically found in Eurofer-97 steel [1-4].
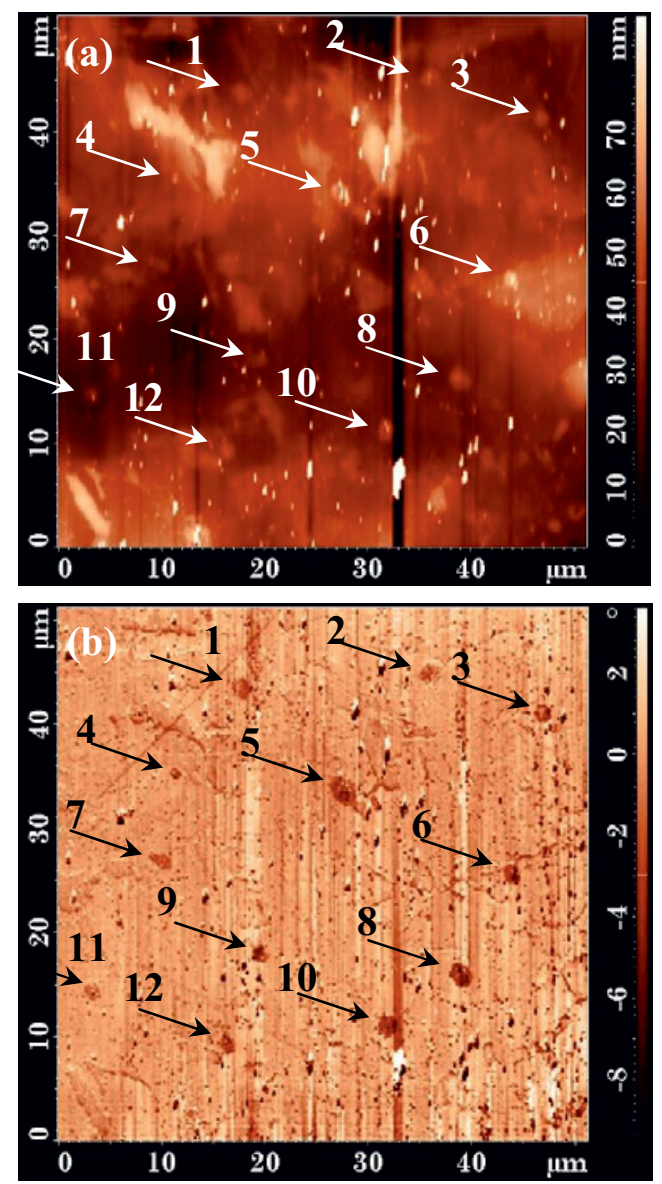

Fig. 3. Representative (a) HS and (b) PS images obtained for the Eurofer-97 steel $\left(50 \times 50 \mu \mathrm{m}^{2}\right)$. The white-numbered arrows of (a) indicate the existence of coarse micrometer SPs and the relative black-numbered arrows of (b) reveal the completely different inelastic properties that these SPs have from the surrounding matrix.

Except for the HS that represents the morphologic topography (relief of the sample surface), during scanning we have simultaneously recorded the PS that relates to the inelastic topography of the specimen [1316]. In figures 3(a) and 3(b) we comparatively present the HS and PS, respectively of an extended $50 \times 50 \mu \mathrm{m}^{2}$ area. Interestingly, some of the coarse micrometer SPs observed in the HS of figure 3(a) (white-numbered arrows), exhibit different contrast from the surrounding matrix in the PS of figure 3(b) (black-numbered arrows). We ascribe the strong PS exhibited by these micrometer SPs to inelastic properties motivated by their different composition from the surrounding Fe-Cr matrix [13-16]. Though we cannot identify the elemental composition of these micrometer SPs, based on electron microscopy data of the literature [1-4] we ascribe them to $\mathrm{M}_{23} \mathrm{C}_{6}(\mathrm{M}=\mathrm{Cr}$, Fe) particles of size $0.1-2.0 \mu \mathrm{m}$. 

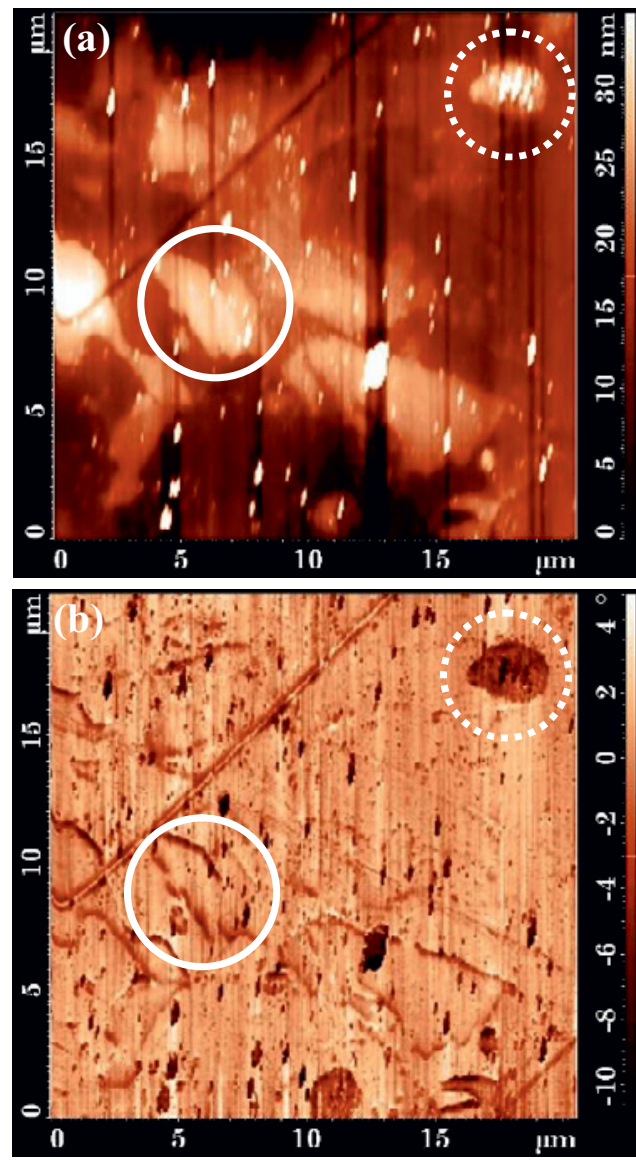

Fig. 4. Representative (a) HS and (b) PS images obtained for the Eurofer-97 steel $\left(20 \times 20 \mu \mathrm{m}^{2}\right)$. The solid and dotted circles highlight two areas that appear to be at the same morphologic level in (a) but clearly exhibit different contrast in (b).

Another example is shown in figures 4(a) and 4(b) for a scanning area $20 \times 20 \mu^{2}$, for the HS and PS respectively. Micrometer areas of the sample surface that appear to be at the same z-level in the morphologic topography of the HS exhibit completely different contrast in the inelastic topography of the PS, as evidenced by the relative solid and dotted circles in figures 4(a) and 4(b), respectively.

The results presented above indicate that Eurofer97 steel exhibits heterogeneous mechanical properties at the micrometer level. This probably originates from heterogeneities in the composition. By comparing the AFM data presented here with the electron microscopy data of the literature [1-4] we speculate that $\mathrm{M}_{23} \mathrm{C}_{6}$ $(\mathrm{M}=\mathrm{Cr}, \mathrm{Fe})$ particles can probably be active sites for the modulation of the mechanical properties of Eurofer-97 steel that is observed upon annealing [1-4].

\section{Conclusions}

The data presented here reveal that AFM can be a trustworthy technique for the study of the morphologic and inelastic topographies of Eurofer-97 steel. Taking advantage of the wide range of dimensions of the scanned area that can be accessed by AFM we investigated not only extended $\sim 50 \times 50 \mu \mathrm{m}^{2}$ areas but also small $\sim 1 \mathrm{x} 1$ $\mu \mathrm{m}^{2}$ areas; micrometer and nanometer SPs were observed accordingly. The nanometer SPs (size below $100 \mathrm{~nm}$ ) can be ascribed to the $\mathrm{TaC}, \mathrm{TiN}$ and $\mathrm{VN}$ fine particles that are typically found in Eurofer-97 steel. Moreover, by utilizing the AFM ability to record the PS, we revealed that the detected micrometer SPs (size up to $2 \mu \mathrm{m}$ ) exhibit inelastic properties that are different from those of the surrounding $\mathrm{Fe}-\mathrm{Cr}$ matrix. These micrometer SPs are ascribed to the $\mathrm{M}_{23} \mathrm{C}_{6}(\mathrm{M}=\mathrm{Cr}, \mathrm{Fe})$ particles of size $0.1-2.0$ $\mu \mathrm{m}$ that are reported in the literature by means of electron microscopy. The present work indicates that Eurofer-97 steel has heterogeneous mechanical properties at the micrometer level. $\mathrm{M}_{23} \mathrm{C}_{6} \quad(\mathrm{M}=\mathrm{Cr}, \mathrm{Fe})$ particles can probably play an important role in the mechanical properties upon annealing, particularly preventing microstructural coarsening.

\section{Acknowledgments}

A. Möslang from the Karlsruhe Institut für Technologie, Germany is acknowledged for supplying the sample.

\section{References}

1. V.B. Oliveira, M.J.R. Sandim, D. Stamopoulos, R.A. Renzetti, A.D. Santos, H.R.Z. Sandim J. Nucl. Mater., 435, 189 (2013)

2. H.R.Z. Sandim, R.A. Renzetti, A.F. Padilha, D. Raabe, M. Klimenkov, R. Lindau, A. Möslang, Mater. Sci. Eng. A, 527, 3602 (2010)

3. P. Fernandez, A.M. Lancha, J. Lape $a$, M. Serrano, M. Hernandez-Mayoral, J. Nucl. Mater., 307, 495 (2002)

4. M. Klimenkov, R. Lindau, E. Materna-Morris, A. Möslang, Prog. Nucl. Energ., 57, 8 (2012)

5. G. Binnig, C.F. Quate, C. Gerber, Phys. Rev. Lett., 56, 930 (1986)

6. H.J. Butt, R. Berger, E. Bonaccurso, Y. Chen, J. Wang, Adv. Colloid Interface Sci., 133, 91 (2007)

7. J.H. Hafner, C.L. Cheung, A.T. Woolley, C.M. Lieber, Prog. Biophys. Mol. Biol. 77, 73 (2001)

8. D. Stamopoulos, V. Gogola, E. Manios, E. Gourni, D. Benaki, D. Niarchos, M. Pissas, Curr. Nanosci. 5, 177 (2009)

9. D. Stamopoulos, E. Grapsa, E. Manios, V. Gogola, N. Bakirtzi, Nanotechnology 23, 485101 (2012)

10. D. Passeri, M. Rossi, E. Tamburri, M.L. Terranova, Anal. Bioanal. Chem., 405, 1463 (2013)

11. A. Schirmeisen, B. Anczykowski, H. Hölscher, H. Fuchs, Dynamic Modes in Atomic Force Microscopy, Chapter 26 in Handbook of Nanotechnology by B. Bhushan (Ed.) ( $3^{\text {rd }}$ Edition, Springer (2010))

12. Y. Song, B. Bhushan, J. Phys.: Condens. Matter 20, 225012 (2008)

13. B. Bhushan, J. Qi, Nanotechnology 14, 886 (2003)

14. J.P. Cleveland, B. Anczykowski, A.E. Schmid, V.B. Elings, Appl. Phys. Lett. 72, 2613 (1998)

15. J. Tamayo, R. García, Appl. Phys. Lett. 73, 2926 (1998)

16. R. García, J. Tamayo, M. Calleja, F. García, Appl. Phys. A 66, S309 (1998) 